ANA ROSA PRATESI

\title{
LA SATURACIÓN DE ESPACIOS DE PODER. TOMADORES DE TIERRAS EN ASENTAMIENTOS DEL GRAN RESISTENCIA (ARGENTINA)
}

La autora es psicóloga, master en epistemología (UNER, Argentina), doctoranda en antropología social (UNaM. Argentina) y docente e investigadora de la UNNE, Argentina.

RESUMEN. Se trata del estudio de un caso de ocupación de terrenos en la ciudad de Resistencia, Provincia del Chaco, Argentina. Se realizó mediante observaciones, entrevistas a informantes calificados y artículos de la prensa local. Se indaga en la dinámica de redes y, en particular, en las estrategias que posibilitaron un periodo de autonomía de la comunidad obturando el ingreso para la intervención externa desde organismos del estado y partidos políticos. Se pone en relieve la actuación complementaria de dos líderes, uno miembro de la iglesia y otro miembro de un partido de izquierda.

ABSTRACT. THE SATURATION OF POWER SPACES. LANDS INVADERS IN SETTLEMENT OF GREATER RESISTENCIA, ARGENTINA.

This article describes a case of occupation of lands in the city of Resistencia, County of the Chaco, Argentina. It was carried out by means of observations, interviews to qualified informants and articles from the local press. It also explores the social dynamic nets. Particularly analyzing strategies that made feasible community autonomy for a certain period which in turn prevented influences from outer organizations such as state' agencies and political parties alike. The article highlights the role-played by two local leaders, a priest and a left wing activist.

\section{Necesidades y oportunidades}

Mayo de 1998 en Resistencia. Los ríos Paraná y Negro crecen inundando sus márgenes, las lluvias son intensas y constantes y anegan zonas internas de la ciudad. Para muchos la situación es insostenible, urge encontrar un lugar donde vivir; los inundados se suman a los que migran desde las ciudades del interior y a los que viven hacinados.[1] Las 11 hectáreas que quedan delimitadas por las avenidas Soberanía Nacional (límite virtual de la ciudad) y Hernandarias, se recortan como un espacio posible para el refugio.

Una testigo del proceso de asentamiento en estas tierras particulares por un grupo de familias es 'M'. Activa integrante de la iglesia católica, estrecha colaboradora de ' $P$ ', el cura párroco de la Iglesia San Cayetano en el barrio Santa Inés. Así lo recuerda:

Ellos hicieron la primera ocupación el 10 de mayo de 1998, se plantaron los primeros ahí, como se diría usurparon esas tierras. Hubo una o dos familias que no podían pagar más el alquiler, no había forma de sostener eso y fueron buscando un lugar. Vieron esos terrenos esa chacra tan grande, que no estaba alambrada ni nada y se plantaron ahí. Naturalmente atrás de ellos empezaron a venir montones más, era tiempo de inundaciones, de lluvias muy torrenciales, permanentes, en término de 15 días había casi 200 familias.

'C' es un dirigente vecinalista y militante de izquierda, conoció la situación desde el primer momento:

En la ocupación hubo tres vertientes: la inmigración del interior, la inundación por lluvia y el hacinamiento. Es graciosa la anécdota del chico de 15 años que viene a averiguar si el hermano va a quedar o no en el asentamiento, porque si se quedaba él podía ocupar el espacio que dejaba dentro de la casa. 
'M' define la situación oscilando entre un acto de usurpación de la propiedad ajena y un hecho épico propio de pioneros:

\begin{abstract}
Ponían chapas, carpas, Ionas, lo que sea. El asunto era tener un pedazo de tierra y un lugar, pero lo de esta gente, que llama la atención era que eran parejas jóvenes, no es gente adulta, sino gente muy joven que estaba buscando su espacio porque después vinieron otros que vivían con sus padres, con sus hermanos, con sus suegras, y estas parejas buscaban un lugar, propio de ellos.

Hay mujeres que nos contaban que ellas se enteraban de esto y les decían a los maridos, yo me voy, y los maridos les decían vos estás loca, si te van a sacar enseguida. Y ellas se jugaron y vinieron y la mayoría eran mujeres, jóvenes, que ninguna pasa los 30, y estaban ahí de día y de noche cuidando la tierra. Hicieron una patriada muy grande esta gente, muy grande. En esa época estaban hasta acá de agua, impresionante.

A mí me hacía acordar los primeros tiempos, no porque lo haya vivido, cuando la gente llegaba y buscaba sus lugares, un espacio, un lugar donde tenerte, donde poder plantar tu rancho, no importa si es de chapa o de cartón, pero es tu tierra, algo tuyo, el derecho a tener tu lugar.
\end{abstract}

\title{
LIDERES COMUNITARIOS Y EXTRACOMUNITARIOS
}

Entre las 200 familias aparecen aquellos con mayor capital cultural y social, con recursos para la movilización y la organización. Se trata de lo que he delimitado como Nivel social jerárquico (heterogéneo u organizado) de la red comunitaria, con integrantes de la comunidad relacionados directa y complementariamente, con interacciones de reciprocidad desigual. Involucra a las organizaciones sociales comunitarias: comisiones de vecinos para el logro de objetivos comunitarios. Supone un primer grado de diferenciación, en cuanto a responsabilidad, acciones y decisiones, entre sujetos semejantes. (Pratesi, 1999)

\footnotetext{
Después ya surgieron los líderes, al ir llegando se fueron juntando, siempre hay alguno con más capacidad de llevar adelante estos grupos. (M)

A la otra mañana ya era un grupo interesante. Me empiezo a meter y a llamar a asamblea. Ya con la experiencia de La Rubita[2] planteo que había que hacer un cuerpo de delegados con la gente de ahí. Siempre hay dos o tres que se destacan, no como concepción política sino de subsistencia.

Eran dos fundamentalmente, era uno que se llamaba Palacios, y otro que era Eloy, éste muere de un infarto masivo, tenía 23 años. Es el que en la asamblea me dice: " yo quiero hablar con usted ' $C$ ', yo quiero que me ayude, yo quiero hacer un barrio, no una villa." Eloy es el que hace toda la mensura. En un momento pudimos hacer el acto de inauguración de la casa de los Sin Tierra, que era la casa de Eloy. (Carlos)
}

Comienza a ser fundamental la intervención de líderes externos a la comunidad, uno de ellos es 'C', el otro el cura de la parroquia vecina, 'P'. Componen el Nivel político social de la red comunitaria, con integrantes de organismos y programas insertos en la comunidad, relacionados con los pobladores de manera directa, complementaria y de reciprocidad desigual. Se trata de una interacción entre sujetos diferentes (Pratesi, 1999).

Ambos han coincidido en distintos eventos en los cuales se debatía el problema de la tierra. Cuenta Carlos:

Se empiezan a hacer en la Argentina encuentros de la Teología de la liberación, el primero se hace en Neuquén. Empiezan al poco tiempo de la democracia, se hacen unos 11 encuentros, en uno de esos me invitaron, en Santiago del Estero, me invitan a mí, yo invito a la cúpula de la Federación de entidades vecinales y vamos.[3]

A mí me invitan porque yo estaba con el tema de la organización de desocupados, que se había decidido hacerlo en forma territorial.

En ese seminario de Santiago lo conozco a ' $P$ ' y a la gente que iba con él, 40 y pico de jóvenes de su parroquia. Había 5000 tipos, de los cuales el 80\% eran jóvenes, un debate intensísimo, que a mí me llamó mucho la atención, se quemó banderas norteamericanas, 


\begin{abstract}
se hace todo el seminario con la metodología de Freire. Había 100 y pico de comisiones, se tocan 80 temas diferentes, ahí surge la idea de articular nacionalmente con estos grupos, había un sector mayoritario en el seminario que se oponían a orientar los seminarios hacia una política definida porque iba a dispersar el movimiento. Es decir siempre y cuando no se toque el sistema, tenían la tesitura de armar la red social, de no meterse mucho en política, porque eso iba a ser tomar posición, se iba a disgregar. La experiencia dice que esa tesitura era la que disgregaba, los seminarios posteriores empezaron a disminuir y al último van 1500 , de lo que sería la izquierda católica. Es el único cura de todo el Chaco en esa tendencia, con la particularidad que se estaba yendo, él quería quedarse pero el obispo no. Él empieza a asumir mayores compromisos.
\end{abstract}

' $M$ ' reconoce con reparos la importancia de la intervención de 'C', al mismo tiempo que sobrevalora la presencia de concejales y diputados. Se trata del Nivel político de la red formado por instituciones, programas, partidos políticos, cultos, que despliegan políticas hacia la comunidad, relacionados en forma indirecta y complementaria con los pobladores. (Pratesi, 1999)

Así fue acompañando Patricio con otra gente que está en la política, que se arrimaba también. Estaba el Concejal M, C ayudó bastante, en la parte organizativa, el tipo tiene, claro tiene sus intereses propios, no... Después también el diputado G, un hombre con mente muy abierta. Entró la comisión de los Sin Tierra, ellos organizaron también.

Sin embargo, mientras ' $C$ ' tiene la iniciativa de apoyar desde el primer momento, ' $P$ ' se acerca a los tomadores cuando es convocado como una estrategia del grupo para su propio fortalecimiento.

Cuando se hace la toma y está la gente ahí, yo digo llamemos al cura, aquí hay un cura en esta parroquia que nos va a acompañar, legitimemos con una misa acá, no en la iglesia. Se hace la misa y le pido la parroquia para hacer las reuniones, y él nos da para todas las reuniones del consejo de vecinos. (C)

A ' $P$ ', lo buscan, él me invita a mí a ir, no me voy a olvidar porque llovía!! ...Lo busca la misma gente de ahí, se arrimaron políticos y gente que trabaja en estas cosas comunitarias. (M)

\title{
ORGANIZACIÓN Y LEGITIMACIÓN DEL MOVIMIENTO
}

Ambos líderes corporizan diferentes experiencias y expectativas.

Cuando se hace la asamblea, yo les empiezo a contar la experiencia de La Rubita, que la gente estaba dividida en tres partes: los radicales, los peronistas y los sin partido y eso fue lo que llevó al desalojo.

Yo me doy cuenta que él $(P)$ empieza a hacer una nueva experiencia donde tiene que poner en práctica mucho de sus discursos y fundamentalmente empieza a ver las contradicciones entre su discurso y el discurso del poder. (C)

Inmediatamente se plantea la organización de la comunidad, reconociendo el Nivel social homogéneo (o de base) de la red comunitaria con integrantes de la comunidad, sujetos semejantes, en relación directa, simétrica, con reciprocidad idéntica y objetivos comunitarios. (Pratesi, 1999)

Les habló de la necesidad de formar un cuerpo de delegados, no de una comisión, sino de un cuerpo horizontal, donde cada uno no representara más de 15 o 20 familias, porque la responsabilidad es muy grande y al no tener experiencia eso iba a (...) Se hizo un primer cuerpo de delegados que empezó a funcionar en la parroquia. (C)

Fuimos conociendo a uno, a otro, distintas formas de pensamiento, también de vivir.

' $P$ ' fue como ayudando a organizarse, yo por lo menos he estado con él en las reuniones, él fue el que les dijo que fueran buscando delegados por manzana, no uno solo, sino 3 ó 4 que representaran una manzana, un sector y que ya había 365 familias que están ahí. (M)

Los tomadores tienen un proyecto para su espacio: 
Eloy dijo "si hacemos de esto una villa nunca más vamos a poder vivir". Bueno fenómeno, eso es fácil vamos a convocar para hacer la subdivisión siguiendo las calles del San Cayetano y las del costado, se hizo más o menos un amanzanamiento, y ahí se dijo a todo el mundo que tenía derecho a un lote de 10 por 20. (C)

Para 'M' ese proyecto fue fruto del convencimiento por parte del cura y sus colaboradores:

Nosotros decíamos que no dejaran entrar más gente, para que no se convirtiera en una villa, que eso fuera un barrio. Y les decíamos ¿qué querían, un barrio o una villa?, una villa es un montón de pasajes, ranchitos por todos lados, un barrio es distinto tiene calles, va a tener escuelas, todas esas formas... (M)

Sin premeditación los hechos se desarrollan con una división de tareas entre los dos líderes, en la cual cada uno despliega las propias fortalezas que complementan a las fortalezas del otro:

\begin{abstract}
Naturalmente se fue dando una división del trabajo: el cuerpo de delegados que manejaba la cuestión política y un grupo "Techo", de solidaridad para construir la casa de la gente, eso se manejaba desde la iglesia. Ahí cada uno en su área, sin llegar a discutirlo se daba naturalmente. (C)

Dos cosas nos preocupaban, uno, que los pobladores no eran usurpadores, que tenían un derecho legítimo, y de eso el cura no sabe nada, ahí bajábamos línea nosotros. Y el cura maneja fundamentalmente la cuestión solidaridad, y después una cuestión interesante que yo descubro, que fortalece subjetivamente a la gente en la lucha contra la represión. En dos o tres oportunidades tuvo una posición de apoyar la resistencia, hubo que resistir una semana contra el asedio de la policía y él entraba, no se permitía pero él entraba con cosas para la gente rompiendo el cerco. (C)
\end{abstract}

Mientras ' $C$ ' era el referente para las decisiones organizativas y políticas, ' $P$ ' lo era para las tareas solidarias a la vez que, a través de su presencia y discurso, legitimaba simbólicamente el hecho de la toma.

\title{
LA SATURACIÓN DE LOS ESPACIOS DE PODER
}

Esta combinación produjo una sinergia grupal que posibilitó una de las condiciones fundamentales para el logro del objetivo comunitario: el bloqueo a otras intervenciones políticas saturando los espacios de poder. "(...) en el fondo no existe Un poder, sino varios poderes. Poderes, quiere decir, formas de dominación, formas de sujeción que operan localmente, por ejemplo, en una oficina, en el ejército, en una propiedad de tipo esclavista, o en una propiedad donde existen relaciones serviles. Se trata siempre de formas locales, regionales de poder, que poseen su propia modalidad de funcionamiento, procedimiento y técnica. Todas estas formas de poder son heterogéneas." (Foucault, 1993: 56)

Se suscitó una lucha de dos o tres meses, para que no se traslade la cuestión de los partidos, que la gente pudiera organizarse en función de sus propios objetivos y con un apoyo que le diera legitimidad.

En una de las asambleas, había dos cosas que había que dar vuelta: que la gente sintiera que lo que estaba haciendo era legítimo y sellar el pacto de silencio en la organización. Se lleva por primera vez la experiencia en el Chaco, nadie sabe como se llama el que está al lado, nadie entrega el nombre de nadie. Era una medida necesaria para evitar el control social y la represión. (C)

Había muchas cosas que pedir, especialmente primero la tierra, la tierra, para que ni la policía ni las autoridades los desalojaran. Tenían que ir organizándose para poder proteger este derecho, esta patriada que hicieron ocupando la tierra. (M)

En el primer año del asentamiento no había políticos. (M)

Los intentos de intervención externa se presentaban como variaciones de un mismo argumento: la ayuda solidaria. 
Hubo una lucha muy fuerte, viene un sector del peronismo a hacer un relevamiento para hacer un comedor, vinculados al duhaldismo. [4] El comedor era sólo para los domingos, ahí hacemos una asamblea para denunciar la trampa.

El segundo ataque viene del diputado $M$, diciendo que él necesitaba el relevamiento, sino no podía hacer la expropiación de la tierra, le tengo que explicar que eso es mentira. Hay que decir cuantas familias hay y nada más. Después les muestro el pedido de expropiación donde no aparecen los nombres. 'M' releva unas 60 familias sobre 400 , no más.

También aparece un médico que filma al cuerpo de delegados y propone un relevamiento para instalar un centro de salud; no trae ningún proyecto ni organización que lo respalde. Cuando se pone en duda sus intenciones desaparece y no vuelve.

Algunos pobladores, preocupados por robos que se habían producido en el asentamiento, piden vigilancia policial. Nuevamente en una asamblea se logra que se desista de ese pedido y se conforme una policía interna con un grupo que patrulle el lugar.

Definimos a la red comunitaria como el sistema relacional de soporte mediante el cual, los individuos y grupos humanos producen y reproducen sus condiciones de vida. En ese sistema los sujetos establecen relaciones directas o mediadas, adjudican distintas jerarquías a los integrantes de la red y establecen interacciones de reciprocidad con el intercambio de bienes, servicios, favores, etc. (Pratesi, 1999)

\section{Las tác ticas del pastor}

El trabajo de Patricio y sus colaboradores se desarrollaba en distintos niveles de la red comunitaria y con actividades diferenciadas. Por un lado organizó un equipo de trabajo integrado por algunos líderes de la comunidad y colaboradores de la iglesia, la función de este equipo estaba relacionada con la elaboración de proyectos y administración de los recursos materiales que se conseguían.

Desde la parte nuestra de la iglesia, llegó el proyecto Techo, que llegó de la Caritas española a la Caritas nacional y a la parroquial, ' $P$ ' agarró. Él va armando como un equipo, lo pone a 'A' como un técnico, llegaron chapas, tirantes para dar, pero él no es que tuviera la idea de dar las chapas así nomás, sino que fue trayendo a la gente, era para 60 familias.

Pero ' $P$ ' se estaba yendo, toma la opción de dejar la parroquia porque estaba buscando otros caminos, y forma un equipo donde entran ' $H$ ', ' $P$ ', ' $J$ ', ' $A$ ', gente que vive en el 10 de mayo, también de la Neike chamigo,[5] este es un proyecto que teníamos antes nosotros, con gente que está asentada en tierras que no son de ellos y no entraron en el lote. Habíamos formado un proyecto Neike para poder hacerles sus casas, el trabajo nuestro era poder solucionar el problema de estas 3, 4 familias.

' $P$ ' forma el grupo Techo, de los cuadros más destacados él los convoca para la solidaridad.

Convoca a un grupo de 60 familias, posibles destinatarias de los recursos, con quienes organiza estructuras de solidaridad con la modalidad de talleres. Esto significa el inicio de una diferenciación, que posteriormente se profundizará, entre los miembros de la comunidad.

Congregó a la gente en la capilla, hacía pequeños talleres, hacía que entre ellos mismos se fueran conociendo, viendo sus necesidades, a ver como se podía empezar y al entregarle las chapas no se le daba a cada familia, sino que se formaban grupos de 4 familias, esas 4 familias se tenían que ayudar a construir el techo, sino se les retiraba, los 4 tenían que ayudarse entre sí, ese era uno de los reglamentos, saber trabajar juntos, que surgió de los talleres. (M)

Los talleres. Primero empezó con que se conocieran entre ellos, la fraternidad, como vinieron así injertándose ... después por qué vinieron ahí, que los trajo a hacer lo que hicieron, esta patriada de agarrar tierras que no eran de ellos, después era sus vidas, la historia personal de cada uno, las historias similares que había y después tratar de hacerles entender que no iban a tener las cosas porque sí nomás, sino que tenían que 
aprender a vivir y a trabajar comunitariamente, no individualmente, ese fue el proyecto. (M)

Fraternidad, identidad y comunidad eran los valores ideas[6] que se inducían a través de esos talleres. Nadie quedaba al margen de su actividad de pastor.

' $P$ ' era un hombre que recorría, que no se quedaba, él con su bicicleta iba y venía al barrio, visitaba a las familias, se sentaba en el suelo, tomaba mate, no tenía ningún tipo de problema, compartía con ellos tal cual.

Quedaron muy enganchados con la imagen de ' $P$ ', no pueden aceptar la imagen de otra cura, aunque nosotros sabemos que no se sirve al hombre sino a Jesús. Lo veían a ' $P$ ' como el profeta, ese tipo que se lo puede mirar, la mayoría no es de la religión católica, ni idea de iglesia. (M)

La actividad religiosa de ' $P$ ' se caracteriza porque escapa a la normativa tradicional de la iglesia, la informalidad acerca a los jóvenes.

Su misa no es la tradicional, es una celebración de la palabra, en realidad es una arenga política con el respaldo del saber divino y ahí él juega un rol importante y yo pienso que ahí él descubre su rol. (C)

Las acciones de ' $P$ ', que no estaban bien vistas por la estructura eclesiástica cuyas autoridades habían decidido un nuevo destino para él, nos recuerdan al análisis que Haley (1994) hace de las innovaciones que introduce Jesucristo: un hombre que ingresa en un escenario de descontento; aislado por la jerarquía religiosa; que se apartaba del discurso y el estilo ortodoxo; forma una organización con hombres capaces de dirigir a otros y se apoya en la misma comunidad en la que se propone influir.

\section{LAS TENSIONES INTERNAS}

La heterogeneidad de los grupos en cuanto a ideología, expectativas, intereses se manifestaba en conflictos que surgían a partir de situaciones puntuales. Los conflictos se daban en torno al polo confianza-desconfianza hacia los funcionarios de gobierno y otros representantes del poder político.

Es el primer encontronazo con el cura. Él dice si no podemos llevar el nombre de la gente no nos van a dar ayuda. Digo no ' $P$ ', el relevamiento no es para ayudar, es para control social y represión. Después viene la citación individual a la gente ante la comisaría o el juzgado, la gente no tiene experiencia, se asusta y se disuelve la organización. Hay que hacer de tripas corazón y aguantar lo que sea, tenés que conseguir lo que puedas sin padrón.

Él entiende y decide no hacer el relevamiento.

Cuando ' $P$ ' ya no está en su parroquia se debe decidir acerca del reparto de ladrillos que habían sido donados para la comunidad.

Se consiguió, hubo problemas, no fue tan fácil. Después llegan los ladrillos, no sé si eran 40 o 60 mil, pero de las fundaciones de San Luis y de otros lados llegaban ladrillos para ayudar a esta gente.

Y bueno hubo dificultades bastante grandes de sortear, como que los ladrillos se habían -yo eso todavía no lo entiendo- que porque se bajó ladrillos, eran dueños de los ladrillos, creo que fue un error de ' $P$ ' haber dicho eso desde Buenos Aires, porque si era así, éramos más los de este lado que bajamos que lo que les correspondía a ellos.

Pasaron un mes o dos de esto, la gente del barrio nos empezó a presionar muy fuerte con la entrega de ladrillos. No se había entregado a nadie porque sale que el gobierno iba a dar las viviendas Aipo.[7] Entonces lo que se quería era usar los ladrillos como completamiento de las viviendas, distribuir mejor en función de las viviendas que se iban a bajar, entonces iba a haber más casas para todos. 
Aparte de eso ya el gobierno al comprar las tierras reorganizó las tierras, y si tenían los ladrillos no iban a tener las casas Aipo. (M)

\section{DESENLACE Y PERSPECTIVAS}

El 23 de diciembre de 1998 se realiza el acto por el cual el Gobernador 'R' hace entrega de los certificados de propiedad a los pobladores. Hay discursos por parte del Gobernador y el Ministro de Obras Públicas 'S', y la Celebración de la Palabra por parte de 'P'. Se había previsto que hablara uno de los pobladores, lo que no sucedió, convirtiéndose en un acto político exclusivo del gobierno, con el silenciamiento de la comunidad.

El logro del objetivo de la propiedad de la tierra por parte de sus pobladores fue el punto de inflexión a partir del cual la comunidad comienza a desmovilizarse. El Lote 133 ya es el Barrio 10 de Mayo.

A la hora de evaluar los hechos los protagonistas hacen pesar aspectos diferentes, los mismos que los habían separado durante todo el proceso.

Es una mezcla de éxito y derrota. Éxito porque están, pero derrota porque no sabés hasta que punto tomaron conciencia. Estaban tocando el punto neurálgico del sistema que es la propiedad privada, eso no se lo podían perdonar objetivamente. Era una lucha muy desigual.

Cuándo me encuentro con ellos tienen cierta vergüenza, me dicen ¿cuando va a venir? Hablando con ellos dicen que han hecho la experiencia más importante de toda su vida. Creo que no tienen conciencia de lo que han hecho. En todas las asambleas, les decía ¿ustedes se dan cuenta de lo que significa para el gobierno, el municipio, etc., lo que están haciendo que se apropien de 10, 12 has. de tierra? (C)

No obstante el proceso no está cerrado, en tanto hay una situación abierta a nuevas alternativas.

Están las condiciones para removilizar. Hay que ir instalarse y crear una escuela de cuadros sociales. Eso hay que hacerlo desde el primer día, ahora lo sabemos para nuevas experiencias. (C)

Ya no es posible sostener el bloqueo a intervenciones partidarias, los espacios de poder cambian de mano.

Cuando el gobierno compra las tierras, ahí sí, el gobernador dijo esto es mío. Vinieron a regalar cosas, fue de terror, tiraban los juguetes desde la camioneta, como si fueran chanchos. Siete meses de trabajo nos tiraron abajo con su circo, la gente quería sus tierras, sus certificados, vinieron las promesas, las cajas, los comedores.

Avanzaron en el barrio los evangélicos, antes ni siquiera intentaban, al haber la fractura del grupo ingresaron. (M)

Recorriendo el barrio se puede ver la proliferación de carteles de propaganda del partido de gobierno, locales del mismo partido y templos evangélicos.

Revisando las promesas suman más las que no fueron cumplidas.

Ahora vemos que ya empiezan a pedir porque ni el "Trabajar"[8] funciona.

Las casas Aipo no se hicieron todavía, el gobierno les dijo hasta el año que viene. Lo que sí, le pusieron el agua y para la luz tienen que tener 100 pilares hechos para que la empresa de energía eléctrica les ponga la luz. ¿Y quién va a poder? Aunque hay algunos avivados, gente que vendió, quedan pocos de los originales, de los que hicieron la patriada y los más pobres son los que menos se oyen, porque ellos saben que no van a poder llegar, no se escucha la voz de ellos.

Diagramaron las calles, pusieron los caños para el agua. (M)

La organización que comenzó a formular el cura no tuvo continuidad. 
Patricio estuvo hasta el 30 de diciembre, él tenía la ilusión de que el equipo pudiera continuar, pero como era nuevo, no tenía raíces todavía, no se pudo continuar. Faltaba que el equipo afiance sus fuerzas, tanto sea en confraternidad como en conocimiento de cada uno de nosotros, y como estaba integrado por diferentes clases de personas, fue muy difícil mantener la unidad. (M)

Tampoco ' $M$ ' considera que la situación de la comunidad esté cerrada a nuevos cambios, se inicia un nuevo proceso.

Si nuestra presencia era para discordia, no convenía. Ahora estamos volviendo muy despacio. (M)

El diagnóstico de situación es absolutamente opuesto para uno y para otro. Mientras que para ' $C$ ' la comunidad no adquirió la suficiente autonomía, para ' $M$ ' la comunidad no se comporta de una forma debidamente heterónoma. En el proceso de autonomización, los pobladores habían realizado conquistas importantes, siendo tal vez los puntos más altos dos hechos en particular:

n El reclamo por el agua, los pobladores se movilizaron hacia el edificio de la empresa provincial de agua potable, allí un grupo se encadenó exigiendo la provisión de agua al barrio.

Después cuando el asunto frente a la empresa de agua, ocupamos la calle y lo llamé a ' $P$ ' para que diera una misa acá. Estábamos rodeados de la policía, la gente temblaba, había que convencerla. Cuando llegó el cura nos sentamos todos en el piso, hizo su misa. (C)

Otro tema fue conseguir el agua, todos encadenados. (M)

n La seguridad autogestionada, con la organización de la policía interna que se describió más arriba, evitando el ingreso de la policía oficial. La seguridad lograda por este medio fue brutalmente quebrada cuando un grupo de delincuentes inducidos por agentes del poder agrede a un grupo de jóvenes, lo que derivó en la muerte de uno de ellos y varios heridos.

Por decisión de la estructura de poder, se arma un asesinato. Instalan una familia con antecedentes policiales en el fondo del barrio. Mataron a mansalva, antes se tuvieron que envalentonar tomando. Los heridos quedaron presos, los atacantes siguen libres. Se hizo una movilización frente al juzgado, después los largan.

Después de la muerte quedó el terror, ahí emerge el radicalismo, acompañado con una campaña macartista. (C)

Coincidiendo con un modelo heterónomo de funcionamiento, la concepción de M de la comunidad no contempla que realicen sus propias reuniones.

Ahí es donde nos enteramos que se habían estado reuniendo ellos en el barrio, en pequeños focos, incentivando a la gente para que vinieran a hacernos esta historia, para llevarse los ladrillos, cuando vimos que se venía muy fea la mano, somos gente de rezo y oraciones, la agresividad era terrible, son agitadores sociales algunos de ellos también, los utilizan en sus necesidades.

Hay autoridades que no tienen discusión aunque no logren la adhesión que había inspirado 'P'.

Decidimos hablar con el párroco nuevo Roberto, le explicamos como estaban las cosas, y las pocas personas que estaban del equipo llamamos a las 60 familias de vuelta, ya no venían más al templo. Se los presentó al párroco, porque la parroquia se extendió hasta el barrio, y entonces sí o sí le gustara a quien le gustara había que respetar al párroco, no podía intervenir ningún otro, la parroquia estaba extendida hasta colonia Amanda. Todo el proyecto techo y ladrillos, iba a continuarse según había quedado, buscando la forma de entregarle 500 ladrillos a cada uno. (M) 
Lamenta que la gente no apoye la planificación que había hecho el equipo.

Se continuó yendo, una parte del equipo iba al barrio y se daban los talleres en los distintos sectores, explicándole todas estas cosas, pero hubo un pequeño grupo que no le gustaba. Porque la gente del barrio era como que la misma ansiedad de ellos por tener las cosas, no toleraba la espera y querían todo ya, ya, ya, y todo es para nosotros.

En otro estudio de caso (Pratesi, 1997) hemos encontrado la misma correspondencia entre organizaciones eficientes y comunidad heterónoma.

$\mathrm{P}$, que ha sido trasladado a la Provincia de Buenos Aires, continua relacionándose con algunas personas de la parroquia. Avanzó en su proyecto de volver al Barrio 10 de Mayo, pero las autoridades eclesiales desbarataron el intento.

Él quiso quedarse, la vicaría de él llegaba hasta Soberanía, va a Buenos Aires y consigue con los pasionistas el apoyo para hacer una parroquia del otro lado, en el barrio. El obispo decide que la vicaría termine en Santa Fe más o menos, para poder entrar tiene que pedir permiso. (C)

\section{CONCLUSIONES - REFLEXIONES}

Desandando el camino recorrido es posible ensayar algunas respuestas a la cuestión que se plantea acerca del funcionamiento de las redes comunitarias y la dinámica que emerge de la relación e intersección de los distintos niveles.

Es fundamental, para quienes aspiramos a vivir en una sociedad compuesta por personas autónomas con capacidad real de decidir y actuar, conocer cuál es el efecto que producen en las comunidades las intervenciones políticas.

Tenemos, por un lado los niveles sociales de la red que se integran alrededor de objetivos comunitarios:

n El de base u homogéneo con sujetos iguales de intercambios recíprocos de naturaleza similar,

n El jerárquico u organizado, con sujetos iguales diferenciados a partir de las capacidades que ponen en juego y las responsabilidades comunitarias que asumen,

y por otro los niveles políticos que se conforman con objetivos propios de las organizaciones sociales, políticas o religiosas externas a la comunidad:

n El político social, integrada por sujetos diferentes de los sujetos del nivel social pero con una relación directa, y,

n El político, compuesto por integrantes de instituciones que establecen una relación indirecta con los niveles sociales.

En este caso ha sido decisivo para la consecución de los objetivos comunitarios la intervención de dos agentes diferentes del nivel político social, uno orientado hacia las decisiones políticas y la organización guiado por el horizonte de la autonomía de los pobladores; el otro orientado hacia las tareas solidarias y la construcción de la identidad comunitaria dentro de los límites de la heteronomía.

La combinatoria de las dos estrategias potenció la capacidad de la comunidad y redundó en la saturación de los espacios de poder, evitando la posibilidad de penetración de agentes del nivel político (funcionarios de estado, de partidos con representación parlamentaria, punteros, etc.) que traían un claro objetivo de sujeción de la comunidad en beneficio de los objetivos políticos propios. 
La actual hegemonía del nivel político en la comunidad fue lograda con medidas extremas:

n El otorgamiento de la reivindicación máxima de los pobladores, la propiedad de las tierras, y el consecuente debilitamiento de la tensión propia del esfuerzo continuado durante meses.

n La manipulación a través del terror -para lo que hizo falta un asesinato- con el debilitamiento de la organización lograda, lo que provocaba un distanciamiento con el líder organizador.

n El alejamiento del líder orientado hacia la solidaridad e identidad, para lo cual fue necesario reformar la distribución geográfica de la organización eclesial.

En relación con los dos polos del nivel político social nos cabe la pregunta acerca de la eficacia comparativa de sus estrategias.

En primera instancia aparece la estrategia basada en la heteronomía con una eficacia en el corto plazo, obturando la intervención a través de formas "técnicas". Sin embargo su eficacia se desvanece en el mediano plazo, con el retiro del líder entre sus seguidores surgen los conflictos y no se plantean otra alternativa que la reproducción de relaciones complementarias, es decir basadas en posiciones de jerarquía.

La estrategia basada en la autonomía aparece como un camino más costoso y esforzado, pero más eficaz en el mediano plazo, la obturación de la intervención netamente política permitió una acumulación de experiencia por la cual hoy existen pequeños grupos que continúan organizándose en pos de sus reivindicaciones.

Esta realidad es la que indica la posibilidad de surgimiento de nuevas formas, o resurgimiento de las anteriores, basadas en la autonomía de los niveles sociales de la red comunitaria.

\section{BIBLIOGRAFÍA}

BARRIOS, FERNANDO

1997. "Asentamiento de población en proceso de incorporación al área urbana del Gran Resistencia". En: Actas de Reunión de Comunicaciones Científicas y Tecnológicas, pp 17-20. Corrientes, Universidad Nacional del Nordeste.

1987. Ensayos sobre el individualismo. Madrid, Alianza Universidad.

DUMONT, LOUIS

1993. Las redes del poder. Buenos Aires, Almagesto

1994. Las Tácticas de poder de Jesucristo. Barcelona, Paidos.

FOUCAULT, MICHEL

HALEY, JAY.

PRATESI, ANA.

1997. "De las necesidades a los recursos. Un giro metodológico en el estudio de la pobreza". $1^{\circ}$ Congreso Internacional «Pobres Y Pobreza En La Sociedad Argentina». Universidad de Quilmes, Buenos Aires.

1999. "Interacciones y niveles de redes comunitarias". En: Comunicaciones Científicas y Tecnológicas, pp 85-88. Corrientes, Universidad Nacional del Nordeste.

ROJAS, DELIA.

1997. "Tomas de tierras. Estudio del caso La Rubita, dado en la ciudad de Resistencia". En: Actas de Reunión de Comunicaciones Científicas y Tecnológicas, pp. 33-34. Corrientes, Universidad Nacional del Nordeste.

SILVA, MANUEL

1995. "Los grupos de tarea". En: Morales, Francisco et al, Psicología Social, pp. 747-759. Madrid, Mc Graw Hill.

[1] Las tomas de tierra por parte de pobladores sin capacidad de compra de viviendas o terrenos se han hecho frecuentes en los últimos años en el Gran Resistencia, se estima que el ingreso de población a esta área urbana alcanza a los 8000 habitantes por año. Para esta población no habría "disponibilidad del suelo urbano, entendido 
como porciones de tierra provistas tanto de la infraestructura y el equipamiento básico necesario, como de la regulación catastral requerida oficialmente." (Barrios, 1997).

[2] Uno de los casos más significativos por su magnitud y trascendencia fue la toma de terrenos de "La Rubita". Delia Rojas (1997) lo caracteriza así: "La Rubita es uno de los terrenos que han quedado rodeado de planes de vivienda y asentamientos particulares, los cuales hicieron implosión sobre el mismo. Lo atípico del caso es que no fue un asentamiento paulatino efectuado en forma espontánea, sino que en el transcurso de 24 horas se movilizaron aproximadamente 500 familias. El estado nacional y provincial afirman que se trató de un hecho conspirativo, preorganizado y con fines puramente políticos. La Federación de entidades vecinales y comunitarias del Chaco sostiene que esta circunstancia se generó de manera espontánea como consecuencia de la necesidad real del techo propio."

[3] Neuquén y Santiago del Estero son dos provincias argentinas.

[4] Duhaldismo: Línea interna del partido justicialista que responde al liderazgo del entonces gobernador de la Provincia de Buenos Aires.

[5] Derivado de la lengua guaraní "Fuerza mi amigo".

[6] Clyde Kluckhon señaló el "estrecho lazo existente entre ideas y valores - es decir, en este caso, entre los aspectos cognitivos y normativos". Dumont $(1987: 246,247)$.

[7] Aipo es un programa social del gobierno provincial que integra asistencia en cuanto alimentación, educación y vivienda.

[8] Se refiere al Plan Trabajar que instrumenta el gobierno nacional, en forma de subsidio, para asistir a los desocupados. 